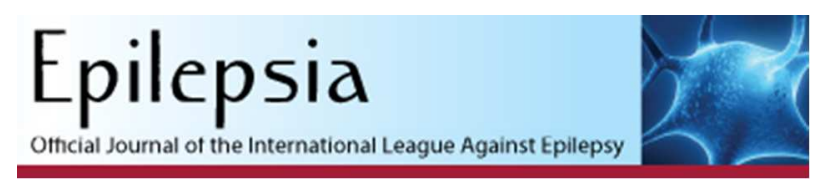

\title{
Individualized prediction of seizure relapse and outcome following antiepileptic drug withdrawal after pediatric epilepsy surgery.
}

\begin{tabular}{|c|c|}
\hline Journal: & Epilepsia \\
\hline Manuscript ID & EPI-00663-2017 \\
\hline Manuscript Type: & Brief Communication (includes Case Reports) \\
\hline Date Submitted by the Author: & 27-Jul-2017 \\
\hline Complete List of Authors: & $\begin{array}{l}\text { Lamberink, Herm; University Medical Center Utrecht, Department of Child } \\
\text { Neurology, Brain Center Rudolf Magnus } \\
\text { Boshuisen, Kim; University Medical Center Utrecht, Department of Child } \\
\text { Neurology, Brain Center Rudolf Magnus } \\
\text { Otte, Willem; University Medical Center Utrecht, Department of Child } \\
\text { Neurology, Brain Center Rudolf Magnus; University Medical Center Utrecht, } \\
\text { Center for Image Sciences, Biomedical MR Imaging and Spectroscopy } \\
\text { Group; Stichting Epilepsie Instellingen Nederland, SEIN, Heemstede } \\
\text { Geleijns, Karin; University Medical Center Utrecht, Department of Child } \\
\text { Neurology, Brain Center Rudolf Magnus } \\
\text { Braun, Kees; University Medical Center Utrecht, Department of Child } \\
\text { Neurology, Brain Center Rudolf Magnus }\end{array}$ \\
\hline Key Words: & prognosis, nomogram, prediction, seizure-recurrence, seizure-freedom \\
\hline
\end{tabular}


Brief communication - for submission to Epilepsia

\title{
Individualized prediction of seizure relapse and outcome following antiepileptic drug withdrawal after pediatric epilepsy surgery.
}

\author{
Herm J. Lamberink ${ }^{1}$, Kim Boshuisen ${ }^{1}$, Willem M. Otte ${ }^{1,2,3}$, Karin Geleijns ${ }^{1}$, Kees P.J. Braun ${ }^{1}$, on \\ behalf of the TimeToStop Study Group*
}

\begin{abstract}
Affiliations:
(1) Department of Child Neurology, Brain Center Rudolf Magnus, University Medical Center Utrecht, Utrecht, The Netherlands; (2) Biomedical MR Imaging and Spectroscopy Group, Center for Image Sciences, University Medical Center Utrecht, Utrecht, The Netherlands; (3) Stichting Epilepsie Instellingen Nederland - SEIN, Heemstede, The Netherlands;

${ }^{*}$ members of the TimeToStop study group are listed at the end of the paper
\end{abstract}

Number of pages: 16 including figure and table

Number of words: $1685 / 1800$

Number of figures: 1

Number of tables: 1

Number of references: $12 / 15$

Supplementary figures: 0

Supplementary tables: 4

Key words: prognosis; prediction; seizure recurrence; seizure-freedom; nomogram 
Individualized outcome prediction after postoperative AED withdrawal in children - manuscript, figures $\&$ tables

Short title: Individualized outcome prediction after postoperative AED withdrawal in children

Corresponding author: Prof. dr. K.P. Braun, Professor of Child Neurology, Division

Neurosciences, Brain Center Rudolf Magnus, Dept. Child Neurology, University Medical Center

Utrecht, room no KC 03.063.0, P.O. Box 85090, 3508 AB, Utrecht, the Netherlands. T: +31 8875

543 41, k.braun@umcutrecht.nl 


\begin{abstract}
The objective of this study was to create a clinically useful tool for individualized prediction of seizure outcomes following antiepileptic drug withdrawal after pediatric epilepsy surgery. We used data from the European retrospective TimeToStop study, which included 766 children from 15 centers, to perform a proportional hazard regression analysis. The two outcome measures were seizure recurrence and seizure-freedom in the last year of follow-up. Prognostic factors were identified through systematic review of the literature. The strongest predictors for each outcome were selected through backward selection, after which nomograms were created. The final models included three to five factors per model. Discrimination in terms of adjusted concordance-statistic was 0.68 (95\% confidence interval [Cl] 0.68-0.69) for predicting seizure recurrence and $0.73(95 \% \mathrm{Cl} 0.72-0.75)$ for predicting eventual seizure-freedom. An online prediction tool is provided on www.epilepsypredictiontools.info/ttswithdrawal [currently only available for review process]. The presented models can improve counseling of patients and parents regarding postoperative AED policies, by estimating individualized risks of seizure recurrence and eventual outcome.
\end{abstract}


Individualized outcome prediction after postoperative AED withdrawal in children - manuscript, figures \& tables

\section{Introduction}

After successful pediatric epilepsy surgery a pressing question for many patients and their caretakers is whether and when to reduce antiepileptic drugs (AEDs). Being able to discontinue drugs is the ultimate proof of successful epilepsy surgery. Stopping medication will prevent further drug adverse effects and may improve cognitive domains, such as psychomotor speed, executive functions and $\mathrm{IQ}^{1-4}$. The decision to withdraw AEDs is partially based on an assessment of the risk of seizure recurrence. Predictors of seizure recurrence on a group level have been identified in previous publications ${ }^{5-10}$, but prediction of relapse is not yet possible for an individual patient.

The TimeToStop study previously showed that early withdrawal unmasks surgical failure but is not related to long-term seizure outcomes ${ }^{6}$. This conclusion was based on multivariable survival analysis, but did not yet enable individualized prediction of AED withdrawal outcomes. We now use the TimeToStop data to create nomograms and a web-based tool to enable estimation of individual risks, analogous to the recently developed prediction models for non-surgical patients $^{11}$. 


\section{Methods}

Data of 766 patients were available from the TimeToStop study; for an extensive description of data collection and definitions we refer to the earlier report ${ }^{6}$. In short, patients were retrospectively identified within 15 pediatric epilepsy surgery centers in Europe. Patients were included if they had epilepsy surgery between 2000 and 2008, were younger than 18 years at surgery, had at least 1 year of follow-up after surgery and if postoperative AED withdrawal was initiated. Data were collected on clinical, surgical and seizure outcome parameters.

Two outcome measures were used: first, seizure recurrence after start of AED withdrawal and second, the seizure status at final follow-up with favorable outcome being defined as seizurefreedom (including freedom from auras) for at least one year at final follow-up. We decided not to model for 'cure' (seizure- and AED freedom at final follow-up), which was the third outcome measure in the original TimeToStop study, because the decision to completely discontinue medication is dependent on the policy of the individual physician, and these policies may change over time or may already have changed. A systematic review on risk factors of postoperative AED withdrawal previously identified 12 potential factors predictive of outcome $^{12}$, listed in supplementary Table 1 . These factors were all available in the TimeToStop dataset.

Missing data were analyzed and imputed through multiple imputations using predictive mean matching. Heterogeneity between centers was tested, and when present, random-effects modeling was used. Assumptions were tested for continuous variables; if the linearity assumption was violated restricted cubic splines with three knots were used. Proportional 
Individualized outcome prediction after postoperative AED withdrawal in children - manuscript, figures $\&$ tables

hazard (Cox) regression analysis was used to account for different follow-up duration. For seizure recurrence, censoring time was the time to seizure recurrence. For seizure-freedom, censoring time was time to seizure recurrence, only for those with unfavorable outcome. All patients with favorable outcome were censored at maximum follow-up. To create a useful set of predictors, the Akaike Information Criterion was used for backward selection of strongest predictors.

For the purpose of the prognostic models, variables with three or four items of which only one was significantly different from the reference category were dichotomized, so that the nonsignificant categories were collapsed with the reference category. This was done for magnetic resonance imaging (MRI; multifocal MRI abnormalities versus focal MRI abnormality and normal MRI), for pre-withdrawal electroencephalography (EEG; interictal epileptiform discharges versus no such discharges or no EEG performed) and for completeness of resection (incomplete resection of the anatomical lesion versus complete resection or completeness not determined). Within the nomograms, risk calculation of seizure recurrence was enabled for two and five years after start of AED withdrawal, and calculation of eventual seizure-freedom was set at six years. We chose six years after start of AED withdrawal to model the chance of eventual "long-term" seizure freedom, creating an one-year longer interval than the latest calculated 5-years relapse risk, as this intuitively allows for an extra period of time to regain seizure-freedom after restart of medication should a late recurrence have occurred.

Internal validation was performed by determining the concordance statistic for each model, corrected for overfitting using 200 bootstrap-samples. Plotting the predicted probabilities 
Individualized outcome prediction after postoperative AED withdrawal in children - manuscript, figures $\&$ tables

against observed proportions of the outcomes was used to visually assess calibration of the separate models.

Analyses were performed with R Statistical software version 3.3.2, using the packages 'MICE', 'coxme' and 'rms'. Ethics approval was obtained for the original TimeToStop study. 
Individualized outcome prediction after postoperative AED withdrawal in children - manuscript, figures \& tables

\section{Results}

One percent of data were missing and imputed (suppl. Table 2). The seizure outcomes after AED withdrawal were: seizure recurrence in $95 / 766$ patients (12\%), seizure-freedom in the last year of follow-up in 733/760 patients (96\%). Median follow-up after start of AED withdrawal was 39 months (interquartile range 21-67 months) (suppl. Table 3). Univariable relations between potential predictors and outcomes are shown in supplementary Table 4.

One of the parameters, the presence of postoperative seizures (excluding seizures in the first two postoperative weeks), was not included in the analysis because none of the 13 cases experienced seizure recurrence and consequently no parameters could be estimated. The median time to reduction for these patients was 24 months (IQR 19-37), compared to 13 months (IQR 6-24) in the patients without postoperative seizures. AED status at final follow-up was known for 11 of these: six patients successfully discontinued AEDs, five were still using AEDs.

The strongest predictors were selected through backward selection, resulting in two models. Table 1 shows the outcome of multivariable Cox regression of these models. Figure 1 is the visualization of the models in the form of nomograms. The predictors for each model and model parameters are listed below. Heterogeneity between centers was low and a regular (fixed-effects) Cox regression was performed. The nomograms were converted to a web-based tool, accessible at $<U R L>$ [sub site not yet accessible for wider public but for the review process accessible at www.EpilepsyPredictionTools.info/ttswithdrawal]. Calibration of the models is visualized in Figures $1 \mathrm{C}$ and $\mathrm{D}$. 
Individualized outcome prediction after postoperative AED withdrawal in children - manuscript, figures $\&$ tables

Factors predicting seizure recurrence were: (higher) age at withdrawal, (shorter) time to AED reduction, multifocal $M R I$ abnormalities, interictal epileptiform discharges on $E E G$, and incomplete resection of the anatomical lesion (Table 1, Figure 1); these factors combined into a prediction model had an adjusted concordance-statistic of 0.68 (95\% $\mathrm{Cl} 0.68-0.69)$.

Factors predicting seizure-freedom at final follow-up were: seizure frequency before surgery, (lower) number of AEDs used at time of surgery, and complete resection of the anatomical lesion (Table 1, Figure 1); these factors combined into a prediction model had an adjusted concordance-statistic of $0.73(95 \% \mathrm{Cl} 0.72-0.75)$. 


\section{Discussion}

We developed a prognostic tool to predict the seizure outcome after AED withdrawal in children who became seizure-free through epilepsy surgery, based on preselected factors that were identified through systematic review of the literature. The nomograms and the web-based tool, developed on a dataset from 766 children, are clinically useful when considering AED withdrawal in an individual patient. The predicted outcomes can serve as a basis to counsel children and caregivers when balancing the risks and benefits of AED withdrawal. The selected predictors are different between the two models. Interestingly, four predictors of seizure recurrence were not predictive of eventual seizure outcome: the timing of AED withdrawal - as revealed in the original TimeToStop study report ${ }^{6}-$, the findings of pre-operative MRI, the age at withdrawal and postoperative EEG abnormalities. Two factors were only related to eventual seizure outcome: the pre-operative seizure frequency had a u-shaped relation with seizure outcome, with the lowest and highest seizure frequencies related to lower chances of eventual seizure-freedom, and the number of AEDs before surgery which showed a linear relation with the outcome. The only factor predictive of both outcomes was the completeness of resection: incomplete resection of the anatomical lesion increased the hazard of seizure recurrence by factor 2.5 and decreased the hazard of eventual seizure-freedom by factor 4 .

Model performance in terms of discrimination was best for predicting eventual seizurefreedom, with an adjusted c-statistic of 0.73 . The calibration plot for this model shows that it is able to accurately identify a small proportion with increased risk, where most patients have a predicted chance between $95 \%$ and $100 \%$ of being seizure-free for at least one year at an 
average follow-up duration of six years after start of AED withdrawal. For predicting seizure recurrence, the adjusted c-statistic of 0.68 shows reasonable discriminatory ability of the model.

The current analysis has several limitations. First, although the analysis was performed on a Europe-wide multicenter cohort and bootstrap-samples were used to correct for overfitting, model performance should ideally also be tested in an external validation cohort. Second, one of the preselected potential predictors, 'postoperative seizures', was not tested because none of the patients with this factor experienced seizure relapse. Although no parameters could be estimated, none of these 13 patients relapsed and six out of eleven patients (55\%) were successful at discontinuing AEDs, which is similar to the $48 \%$ in the complete cohort. Therefore, the current data do not indicate an increased risk for patients with postoperative seizures, once it has been decided to withdraw AEDs. However, the time to reduction was almost twice as long for these patients, indicating that they had a substantial longer period of seizure-freedom after the initial post-surgical relapse before reduction of AEDs. Lastly, it is important to realize that postoperative seizure relapse risk has never been compared between patients who do, and those who do not withdraw medication, in a prospective randomized manner. Our data, and all cohort studies reporting on post-withdrawal relapse rates, only included a selection of patients in whom the treating physician deemed it safe to start AED withdrawal. It cannot be excluded that recurrence rates, predicted by the current model, are somewhat higher when applied in a total cohort of unselected children who underwent epilepsy surgery. Therefore, the risk predictor is most reliably applied in patients in whom physicians already consider withdrawal of 
medication, but want to balance its risks and benefits to better counsel the individual patient and his or her caregivers.

In conclusion, two prognostic models with moderate to good discriminative performance were created and converted to a clinically useful tool to predict the individual risk of seizure recurrence and eventual seizure-freedom, for children after epilepsy-surgery.

\section{Disclosures}

We declare no competing interests. We confirm that we have read the Journal's position on issues involved in ethical publication and affirm that this report is consistent with those guidelines.

\section{Acknowledgements}

This study was funded by the Epilepsiefonds.

\section{TimeToStop Study group}

Austria. M Feucht, G Gröppel (Medical University Vienna, Vienna). 
Individualized outcome prediction after postoperative AED withdrawal in children - manuscript, figures $\&$ tables

France. P Kahane, L Minotti (University Hospital Grenoble, Grenoble); A Arzimanoglou, P Ryvlin, E Panagiotakaki, J de Bellescize, K Ostrowsky-Coste (University Hospitals of Lyon, Lyon); E Hirsch, M Valenti (University Hospital Strasbourg, Strasbourg).

Germany. T Polster (Bethel Epilepsy Center, Bielefeld); R Sassen, C Hoppe, S Kuczaty, C Elger (University Hospital, Bonn, Bonn); S Schubert (University Hospital Heidelberg, Heidelberg); K Strobl, T Bast (Epilepsy Center Kork, Kehl-Kork).

Italy. C Barba, R Guerrini, F Giordano (Meyer Children's Hospital, University Hospital, Florence);

S Francione, D Caputo (Claudio Munari Epilepsy Surgery Center Ospedale Niguarda, Milan).

Netherlands. K Boshuisen, K P J Braun, C S P M Uiterwaal, O van Nieuwenhuizen, F S S Leijten, P C van Rijen (University Medical Center Utrecht, Utrecht).

Switzerland. M Seeck (Neurology Clinic, University Hospital Geneva, Geneva).

Turkey. D Yalnizoglu, G Turanli, M Topcu (Haceteppe University Ankara, Ankara); C Özkara, M Uzan (Istanbul Unversity, Cerrahpasa Medical Faculty, Istanbul).

United Kingdom. J H Cross, L D’Argenzio, W Harkness (University College London Institute of Child Health, Great Ormond Street Hospital for Children NHS Foundation Trust, London) 


\section{References}

1. Skirrow C, Cross JH, Cormack F, et al. Long-term intellectual outcome after temporal lobe surgery in childhood. Neurology 2011;76:1330-7.

2. Van Schooneveld MMJ, Van Erp N, Boshuisen K, et al. Withdrawal of antiepileptic drugs improves psychomotor speed after childhood epilepsy surgery. Epilepsy Res.

2013;107:200-3.

3. Boshuisen K, Van Schooneveld MMJ, Uiterwaal CSPM, et al. Intelligence quotient improves after antiepileptic drug withdrawal following pediatric epilepsy surgery. Ann. Neurol. 2015;78:104-14.

4. Helmstaedter C, Elger CE, Witt JA. The effect of quantitative and qualitative antiepileptic drug changes on cognitive recovery after epilepsy surgery. Seizure 2016;36:63-9.

5. Lee $S-Y$, Lee J-Y, Kim DW, et al. Factors related to successful antiepileptic drug withdrawal after anterior temporal lobectomy for medial temporal lobe epilepsy. Seizure 2008;17:11-8.

6. Boshuisen K, Arzimanoglou A, Cross JH, et al. Timing of antiepileptic drug withdrawal and long-term seizure outcome after paediatric epilepsy surgery (TimeToStop): A retrospective observational study. Lancet Neurol. 2012;11:784-91.

7. Park K II, Lee SK, Chu K, et al. Withdrawal of antiepileptic drugs after neocortical epilepsy surgery. Ann. Neurol. 2010;67:230-8.

8. Menon R, Rathore C, Sarma SP, et al. Feasibility of antiepileptic drug withdrawal following extratemporal resective epilepsy surgery. Neurology 2012;79:770-6.

9. Yardi R, Irwin A, Kayyali $\mathrm{H}$, et al. Reducing versus stopping antiepileptic medications after temporal lobe surgery. Ann. Clin. Transl. Neurol. 2014;1:115-23.

10. Berg AT, Vickrey BG, Langfitt JT, et al. Reduction of AEDs in postsurgical patients who attain remission. Epilepsia 2006;47:64-71.

11. Lamberink HJ, Otte WM, Geerts AT, et al. Individualised prediction model of seizure recurrence and long-term outcomes after withdrawal of antiepileptic drugs in seizurefree patients: a systematic review and individual participant data meta-analysis. Lancet Neurol. 2017;16(7):523-531. http://dx.doi.org/10.1016/S1474-4422(17)30114-X.

12. Lamberink HJ, Otte WM, Geleijns K, et al. Antiepileptic drug withdrawal in medically and surgically treated patients: A meta-analysis of seizure recurrence and systematic review of its predictors. Epileptic Disord. 2015;17:211-28. 
Individualized outcome prediction after postoperative AED withdrawal in children - manuscript, figures $\&$ tables

Figures and tables

Table 1. Predicting the outcome of AED withdrawal in seizure-free children after epilepsy surgery.

\begin{tabular}{|c|c|c|c|c|}
\hline & \multicolumn{2}{|l|}{ Seizure recurrence } & \multicolumn{2}{|c|}{$\begin{array}{l}\text { Seizure-freedom at last follow-up } \\
\text { (seizure-free } \geq 1 \text { year) }\end{array}$} \\
\hline & $\mathrm{HR}(95 \% \mathrm{Cl})$ & p-value & HR (95\% Cl) & p-value \\
\hline Time to reduction (months from surgery) & $*$ & $*$ & & \\
\hline \multicolumn{5}{|l|}{ MRI } \\
\hline focal abnormality or normal & Reference & & & \\
\hline multifocal abnormalities & $2.39(1.36-4.22)$ & 0.003 & & \\
\hline Age at withdrawal (years) & $1.06(1.01-1.10)$ & 0.008 & & \\
\hline \multicolumn{5}{|l|}{ Postoperative EEG } \\
\hline normal or not performed & Reference & & & \\
\hline interictal epileptiform discharges & $1.72(1.10-2.68)$ & 0.02 & & \\
\hline \multicolumn{5}{|l|}{ Complete resection of the anatomical lesion } \\
\hline complete or not determined & Reference & & Reference & \\
\hline incomplete & $2.46(1.55-3.39)$ & $<0.001$ & $0.23(0.11-0.51)$ & $<0.001$ \\
\hline $\begin{array}{l}\text { Seizure frequency (number of seizures per } \\
\text { day) }\end{array}$ & & & * & " \\
\hline Number of AEDs at time of surgery & 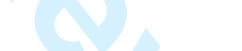 & & $0.68(0.46-0.99)$ & 0.05 \\
\hline \multicolumn{5}{|l|}{ Model performance } \\
\hline Adjusted c-statistic ( $95 \%$ confidence interval) & $0.68(0.68-0.69)$ & & $0.73(0.72-0.75)$ & \\
\hline Percentage optimism adjusted for & $2.8 \%$ & & $4.9 \%$ & \\
\hline
\end{tabular}

Multivariable models with strongest predictors for seizure recurrence and seizure-freedom at last follow-up. Categorical variables with three or four items of which only one was significantly different from the reference category were dichotomized, so that the non-significant categories were collapsed with the reference category.

*not linearly modelled, restricted cubic splines used with three knots. For the effect size, see figure 1.

$A E D$, antiepileptic drug; c-statistic, concordance-statistic; $\mathrm{Cl}$, confidence interval; $\mathrm{EEG}$, electroencephalography; $\mathrm{HR}$, hazard ratio; MRI, magnetic resonance imaging. 


\section{A. Predicting seizure recurrence after AED withdrawal} in children who are seizure-free after epilepsy surgery
1.

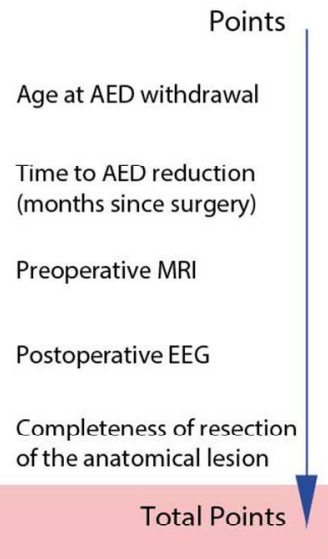

3. Recurrence risk at 2 years

Recurrence risk at 5 years

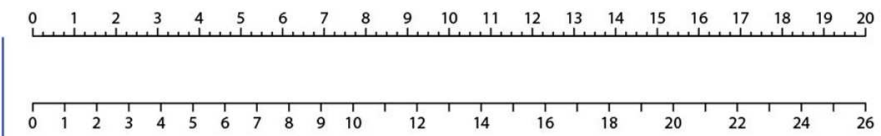

\section{multifocal}
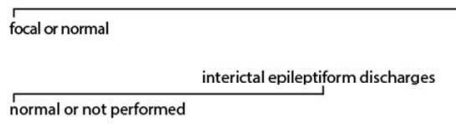

$$
=
$$
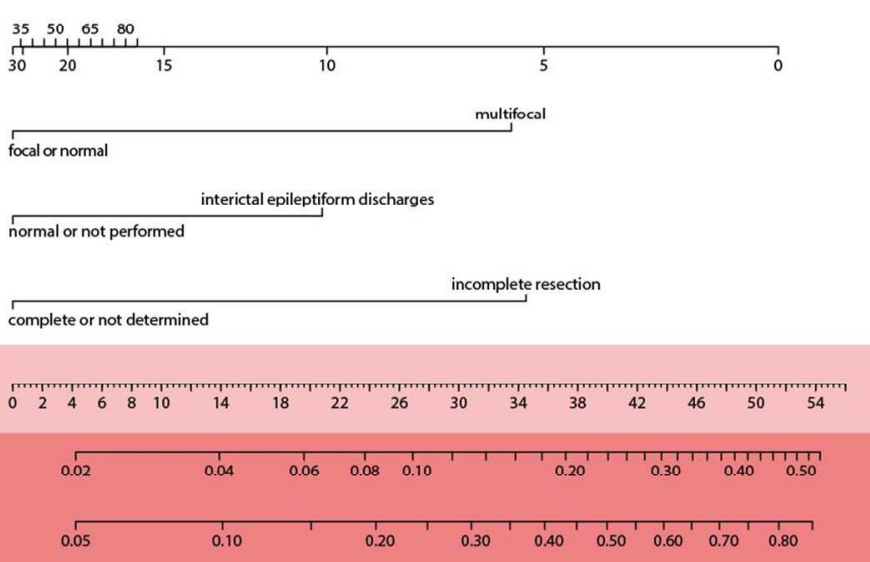

\section{B. Predicting long-term seizure-freedom after AED withdrawal} in children who are seizure-free after epilepsy surgery

1.

Points تصس

Average seizure frequency per day before surgery

Number of AEDs at time of surgery

Completeness of resection of the anatomical lesion

2. Total Points

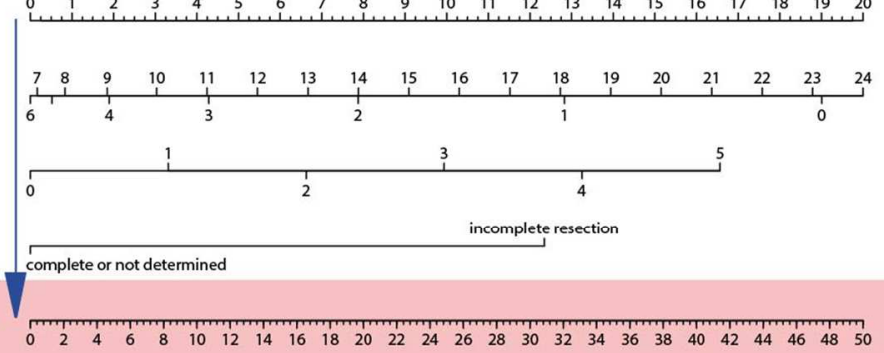

3. Seizure freedom at 6 years (seizure-free for at least 1 year)
$0.80 \quad 0.70 \quad 0.600 .500 .40$

\section{Instructions}

Determine individual risk in three steps:

1. For every variable on the left, count the points given at the top.

2. Add the points to a total score

3. Determine associated risk of seizure recurrence, seizure-freedom and cure

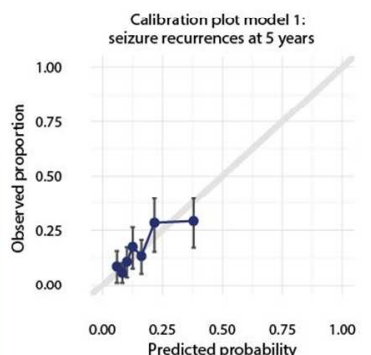

\section{c.}

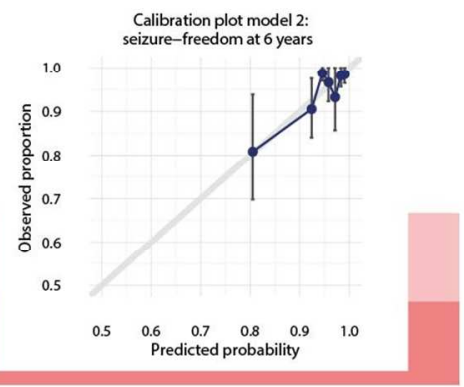

Figure 1. Nomograms predicting the outcome of AED withdrawal in seizure-free children after epilepsy surgery Two nomograms predicting seizure outcomes of AED withdrawal, based on the models presented in table 1. (A) prediction of seizure recurrence in the first 2 and 5 years and (B) prediction of seizure-freedom (seizure-free for at least one year) at six years after surgery. (C) and (D) are the respective calibration plots. Predicted probabilities are plotted against the observed proportions for the 766 patients in the analysis, divided into groups of $k=100$. Alignment on the grey diagonal indicates good calibration, large deviation indicates worse calibration. 